PREHOSPITAL CARE

\title{
Prehospital management and medical intervention after a chemical attack
}

\author{
L Kenar, T Karayilanoglu
}

Emerg Med J 2004;21:84-88

Chemical warfare agents are toxic weapons and emergency prehospital medical care providers should be well prepared, trained, and equipped to give response. Personnel need to be aware of the following medical issues regarding prehospital management of a chemical attack, event recognition, incident medical command and control, safety and protection, decontamination, isolation of the incident area (hot zone, warm zone, and cold zone), sampling and detection, psychological management, communication, triage, treatment, transportation, recovery activities and fatality management. During prehospital response, healthcare responders should provide self protection by wearing proper protective equipment and ensuring that the casualty is thoroughly decontaminated. Medical first responders are also responsible for performing triage in each zone of the incident area. Victims are triaged into four categories based on the need for medical care; immediate, delayed, minimal, and expectant. Finally, a medical emergency planning should be completed, and exercises conducted to test the system before an event occurs.

See end of article for authors' affiliations

Correspondence to:

Correspondence to:
Dr L Kenar, Department of NBC Defence, Gulhane Military Medical Academy, 06018 Etlik, Ankara, Turkey; Ikenar@ gata.edu.tr

Accepted for publication 29 July 2003
C hemical warfare agents (CWAs) are weapons that can be used not only for military purposes, but also on civilian populations as weapons of terror because of their toxicity and rapid effect. ${ }^{12}$ Terrorist interest in the use of chemical and biological (CB) weapons has grown substantially since the Tokyo subway was attacked with sarin, a nerve agent, in $1995 .^{34}$ The recent war between the United States and Iraq as well as the current threat of further US military involvement in the Persian Gulf region has reportedly raised the prospect of the use of $\mathrm{CB}$ weapons both on the battlefield and in terrorist attacks on civilian populations anywhere in the world. There has always been the possibility of a chemical attack, but only since 11 September 2001 have we been especially aware of this vulnerability. In addition to the death and physical injury they cause, the use of CWAs may also give rise to fear, panic, and psychological trauma throughout the population. Fear of the use of these weapons cause the government and the general public to expand resources to tackle this threat, including purchasing protective equipment, delivering specialised training. In many ways, medical preparedness is the best defence and is something that can be done immediately. Successful management of casualties in a toxic chemical attack depends on planning, preparation, and training. Medical care of the casualties depends on knowledge of the agent and timely intervention. ${ }^{5}{ }^{6}$ This article aims to present an overview of the highlights of emergency medical and first aid issues related to preparedness and response against CWAs.

\section{METHODS}

Potential sources of exposure include accidental release from industrial production facilities or stockpiles, direct military attacks, industrial sabotage, and intentional release as an act of terrorism (box 1).

It is widely accepted that prehospital and emergency medical personnel need to be well prepared, trained, and equipped to successfully respond to chemical incidents. These personnel include emergency medical technicians, emergency physicians, and emergency nurses. Emergency medical response personnel need to be aware of the following medical issues regarding prehospital management of a chemical attack $^{5}$.

- Event recognition

- Incident medical command and control

- Safety and personal protection

- Decontamination

- Isolation of the incident area

- Sampling and detection

- Psychological management

- Communication and coordination

- Triage

- Treatment

- Transportation

- Recovery activities

- Fatality management

Some of these points must be taken into consideration as pre-attack measures performed by medical units. Of them, priority should be given to incident site isolation and zone establishment together with triage and transportation-that is, evacuation is a priority, because survival depends on the speed with which the casualty receives definitive care.

Abbreviations: $C W A$, chemical warfare agent; $C B$, chemical and biological 
Box 1 Potential sources for an incident where a chemical weapon can be released

- Crowded places

- Embassies/diplomats' residencies

- Subways

- Governmental facilities

- Airports

- Ceremonies

- Shopping malls

- Universities/schools

- Research/medical facilities

- Amusement parks

- Theatres, movies

- Sport stadiums/arenas

\section{RESULTS AND DISCUSSION}

First of all, the attack must be recognised as a chemical attack, therefore medical responders must be familiar with the indicators of chemical agent use (box 2). Once the incident has been recognised as attributable to CWA use, emergency medical personnel can more easily determine the required intervention. ${ }^{7-9}$

In the case of the medical approach, the zone established must also be prioritised. In a chemical incident, a "hot line" concept is used. People on one side of this clearly demarcated line are considered "dirty" (contaminated), and people on the other side are considered "clean". Initially the hot line should be established upwind of the accident (fig l), but will eventually encompass the entire affected area. ${ }^{8}{ }^{10}$

Anyone, including medical personnel, entering the dirty area must wear appropriate protective equipment (usually respiratory protection and protective overgarment) and must be decontaminated before coming back to the clean area. In the hot zone (fully contaminated zone), established by the detection team who have special CWA detectors like the CAM (chemical agent monitor) (fig 2) and other specialised equipment, the main objective is to evacuate the victims to the clean area as soon as possible. The specific first aid and antidotal treatment can be administered before, during, and after evacuation as the situation dictates.

Timely identification of the chemical agent is critical for the protection of the population. Various detection systems that may be of most use within the medical community exist like chemical agent detection paper that detects and

\section{Box 2 General indicators of possible chemical} agent use

- Unusual occurrence of dead or dying animals (like dead birds).

- Unexplained casualties (multiple victims with the similar signs and symptoms of, for example, skin, respiratory system, and nervous system).

- Increase of the frequency of those with the above signs as to the wind direction.

- Unusual liquid or vapour (droplets, unexplained odour).

- Suspicious dispersal devices or packages (spray devices and munitions).

- Mass casualty without any conventional injuries.

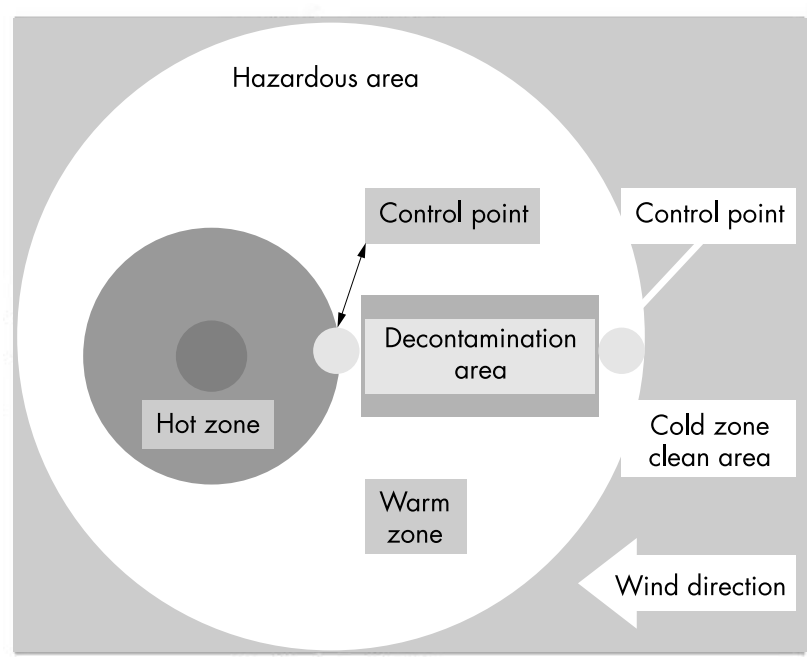

Figure 1 After a chemical incident, the zones where medical intervention differs must be established.

identifies liquid agents that are sensitive to three indicator dyes suspended in the paper matrix. As mentioned previously, CAM is a hand held device using ion mobility spectrometry technology to discriminate between nerve agent vapour and mustard or cyanide in some types. However, attention must be given to false readings on this monitor when used near strong vapour sources such a dense smoke, exhaust gases, and fumes. ${ }^{89}$

Decontamination, which is a special aspect in the response of a chemical incident, is a neutralisation or removal process, or both, performed by application of a decontaminating compound and/or thorough flushing with water, depending on the type of agent. ${ }^{11}{ }^{12}$ Transferring patients from a chemical incident site directly to a hospital without decontamination may be a mistake. Decontaminating the victims correctly at incident scene is of great importance and will prevent healthcare workers becoming unnecessarily contaminated. Assuming patients are generally poorly decontaminated at the incident site, hospitals must be prepared with decontamination facilities, trained decontamination personnel, and a well designed and tested decontamination protocol (box 3). Decontamination is ideally done outside at the hot line in a serial manner of decontamination stations shown in figure 1. A decontamination agent, for example, a $0.5 \%$ solution of hypochlorite (one part household bleach to nine parts water) should be gently applied and rinsed off with water at the last station. That rubbing and scrubbing the skin may enhance the chemical agent absorption must be kept in mind. ${ }^{6}{ }^{10}$

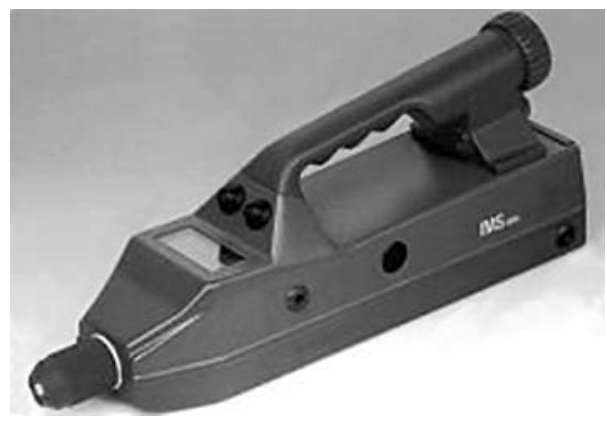

Figure 2 CAM (chemical agent monitor) can be used to detect the released agent in the incident site. 
Box 3 A hospital decontamination protocol specified for each hospital should be organised and practised as a countermeasure against chemical terrorism

- ED staff must be familiar with the protocol

- To choose a decontamination area

- To select a route that will be used to take the patient to that area

- A sufficient warning system

- To obtain adequate information about the chemicals involved, mechanism of contamination, degree of contamination, patient's status and any suspected coexisting injuries or illnesses.

- To specify the members of the decontamination team

- To establish the necessary clean up procedures (rinsewipe-rinse is basically taken as the main procedure)

- To provide all clothes, disposable suits placed in specially marked containers

- To arrange the routes of removal for waste items

In the event of an attack with chemical agents, medical rescuers and healthcare providers must also consider first aid and treatment approaches in the overall evaluation of contaminated casualties.

Vital functions should be aggressively supported and $\mathrm{ABC}$ (airway, breathing, circulation) must be maintained. Antidotal therapy may be concerned for either chemical warfare agent, and it facilitates the ventilation by decreasing the constriction in respiratory system. Early administration of atropine is absolutely vital for the correct management of nerve agent poisoning. Atropine can be given intramuscularly/intravenously at a rate of $2 \mathrm{mg}$ every three to five minutes until the casualty is atropinised. However, in severe cases, between $30-50 \mathrm{mg}$ of atropine in total may be required to achieve atropinisation. Pralidoxime, one of the available oximes, reactivates the inhibited acetylcholinesterase, and intravenous or intramuscular injection of bolus doses of oxime is probably a practical solution under field conditions. The drug treatment recommended for a chemical casualty is agent dependent, and given in table $1^{8-10} 13$

The most important rule for any responder to a chemical event is self protection. There are two basic methods:

- Wearing adequate protective suit, including appropriate respiratory protection.
- Being sure that the casualty is thoroughly decontaminated.

The military protective suit has an interior layer of activated carbon that adsorbs CWAs. The medical staff should wear protective clothing that provides at least level C protection (table 2). ${ }^{2614}$ When the potential exists for splash or immersion by chemicals, or for exposure to vapours, fumes, gases, or particulates that are harmful to skin or capable of being absorbed through the skin, a totally encapsulating non-permeable suit should be used. For first responding health personnel, a permeable protective suit might also be provided during the medical intervention. One of the pieces of protective equipment is the gas mask, which is especially effective against volatile agents. It is important to note that improper fitting of the mask may cause psychological stress and a possible exposure, therefore, frequent exercises and training with this equipment are essential to overcome most of the discomfort and the reduction in performance related to its use. ${ }^{210}$

After a CWA release, a triage station should be established in the warm zone, where decontamination typically occurs, to determine the priorities for resuscitation, decontamination, pharmacological therapy, and transport to hospital. Triage is a dynamic process and should be performed continuously in both hot and warm zones, and be conducted by specially trained medical personnel who know the effects and treatment of CWAs, as well as standard trauma. ${ }^{6-8} 10$

The triage system commonly used by medical units includes four categories that are based on the need for medical care and the resources available; immediate, delayed, minimal, and expectant:

Tl (immediate): casualties who require medical care and advanced life support within a short time on the incident site and in the hospital. Victims thought to be exposed to nerve agents who have moderate to severe effects in two or more systems like respiratory, gastrointestinal, muscular systems are included in this category. Similarly, airway injury in vesicants exposure and unconsciousness, convulsions with or without apnea might be indicated for immediate triage category. Usually, casualties with respiratory rates above or equal to 30 or below or equal to nine breaths per minute and those whose capillary refill is greater than two seconds are $\mathrm{Tl}$.

T2 (delayed): casualties with injuries who are in need of prolonged care and require hospitalisation, but delay of this care does not affect the prognosis of the casualty. If capillary refill is less than two seconds then the patient is assigned to this priority. Patients who are exposed nerve agents and cyanides and survived after antidote administration are

Table 1 Antidotal treatment available for the chemical warfare agents

\begin{tabular}{|c|c|c|}
\hline Agents & Antidote & Dose \\
\hline \multirow[t]{4}{*}{ Nerve agents } & Atropine & $\begin{array}{l}2-8 \mathrm{mg} \mathrm{IM} / \mathrm{IV} \text {. Full atropinisation maintained at } \\
2 \mathrm{mg} \text { doses every } 3 \text { to } 5 \mathrm{~min} \text { for several hours. }\end{array}$ \\
\hline & Pralidoxime & $1-2 \mathrm{~g} \mathrm{IV}(0.5 \mathrm{~g} / \mathrm{min})$ in saline. \\
\hline & Diazepam (valium) & 5-10 mg IV/IM/orally \\
\hline & Pyridostigmine bromide & $30 \mathrm{mg}$ every $8 \mathrm{~h}$. \\
\hline \multirow[t]{2}{*}{ Lewisite } & BAL (dimercaprol) & $\begin{array}{l}\text { Commercial preparation of } \% 10 \mathrm{BAL} \text { in peanut oil } \\
\text { up to } 4.0 \mathrm{ml} \text { IM. Repeat in } 4,8,12 \mathrm{~h} \text {. }\end{array}$ \\
\hline & $\begin{array}{l}\text { BAL analogues (DMPS, DMSA, } \\
\text { DMPA) }\end{array}$ & DMSA $300 \mathrm{mg}$ orally every $6 \mathrm{~h}$ for three days. \\
\hline \multirow[t]{4}{*}{ Hydrogen cyanide } & Amyl nitrite & $\begin{array}{l}\text { Inhaled for } 30 \mathrm{~s} / \mathrm{min} \text { and maintained until the } \\
\text { initiation of sodium nitrite. }\end{array}$ \\
\hline & Sodium nitrite & IV infusion of $10 \mathrm{ml}$ over $3-5 \mathrm{~min}$. \\
\hline & Sodium thiosulfate (25\%) & IV infusion of $50 \mathrm{ml}$ \\
\hline & 4-DMAP & $3 \mathrm{mg} / \mathrm{kg}$ IV injection \\
\hline BZ (incapacitant) & Physostigmine & $2 \mathrm{mg}$ in $10 \mathrm{ml}$ saline IV (over $5 \mathrm{~min}$ ) \\
\hline
\end{tabular}




\begin{tabular}{|c|c|c|}
\hline Level A & $\begin{array}{l}\text { Maximum level of protection and used } \\
\text { in very high concentrations of toxic agents. }\end{array}$ & $\begin{array}{l}\text { Fully chemical protective suit, positive pressure self } \\
\text { contained breathing apparatus, double layers of } \\
\text { chemical resistant gloves and boots. Airtight seals } \\
\text { between the suit and inner layer of face, hand, } \\
\text { and foot protection. }\end{array}$ \\
\hline Level B & Used when the dangers to the skin are less. & $\begin{array}{l}\text { Full respiratory protection similar to level A } \\
\text { excluding airtight seals. }\end{array}$ \\
\hline Level C & $\begin{array}{l}\text { Used when air concentration of the agent is } \\
\text { much lower. }\end{array}$ & $\begin{array}{l}\text { Face cartridge mask, chemical resistant suit } \\
\text { with gloves and boots. }\end{array}$ \\
\hline Level D & $\begin{array}{l}\text { Used only when there is no danger of } \\
\text { chemical exposure. }\end{array}$ & $\begin{array}{l}\text { Latex gloves, eye splash protection, and } \\
\text { no respiratory protection. }\end{array}$ \\
\hline
\end{tabular}

generally assessed in this category. But, the patients with vesicant skin injury $>5 \%,<50 \%$ of body surface area; eye injuries, and the airway problems starting $>6$ hours after exposure can be categorised herein.

T3 (minimal): casualties who have minor injuries who will not be evacuated and will be able to return to duty in a short time. For nerve agent exposure, those can walk and talk and are capable of self aid. Skin injury less than 5\% of body surface and minor eye injuries are also considered for vesicants.

T4 (expectant): casualties with fatal injuries who will probably not survive with medical care available before reaching definitive care. Circulation failed and more than $50 \%$ of body surface is affected because of chemical weapons.

Another point that should be emphasised is that triage is done based on need and resources available for medical or surgical care, not priority for decontamination because all chemical casualties require decontamination. After receiving emergency medical treatment, the contaminated casualties must go through the decontamination stations before receiving further medical care in the clean, or prehospital environment, as detailed in box 4 .

As the post-incident procedure, it should be noted that victims triaged on fourth category or those who have died be

Box 4 Some items that have to be considered in a minimal patient care in the chemical incident site if available

- Enough decontamination (15-30 minutes if available)

- Basic life support measures and lifesaving procedures must be performed in the decontamination area.

- If medically indicated, cardiac monitorisation, intravenous access route, and oxygen support to be administered.

- Conditions such as tension pneumothorax or respiratory distress should be treated immediately.

- Hypothermia should be considered in the case of water decontamination.

- In the case of trauma, if available, blood for haemoglobin determination and cross match should be sent.

- Anything in the decontamination area should be considered potentially contaminated.

- Patient decontamination should be performed by first removing the patient's clothes, this will take care of $70 \%$ to $85 \%$ of the contaminant. After sufficiently washed off, the patient may be moved into the emergency department. decontaminated and recorded for use in forensic investigations and a possible necropsy. A reserved morgue must be established to prevent cross contamination and the burial process should be applied with a great care with the suggestion that the deceased had been involved in a chemical incident.

Detection and diagnosis of illness and injury caused by chemical exposure constitute a complex process that entails the activities of many local and community resources, which can assist in resolving the situation. Therefore, the following organisations should establish a strong collaboration with each other:
- Public health organisations
- Poison control centres
- Medical research centres
- Professional societies
- Emergency response units
- First responder organisations
- Safety and medical equipment manufacturers
- Military organisations
- Intelligence services
- Law facilities.

Some critical information should be ascertained that may be useful for hospital or other local providers. If available, the following data should be obtained and transmitted:

- Number and types of casualties

- Chemical substance involved (if known though often not determined)

- Estimated time of arrival of the casualties at the hospital

- Time of incident and location of incident site

- Method of contamination (vapour or liquid)

- Potential hazards to healthcare providers.

\section{CONCLUSION}

Chemical weapons are still a great threat, for which all medical and healthcare facilities must be prepared. So, medical planning must be done in peace time so that detection and surveillance, laboratory analysis, emergency response and communications are effective in time of need. To achieve this multidisciplinary cooperation, training and preparedness should be provided to at least basic emergency medical assets.

\footnotetext{
Authors' affiliations

L Kenar, T Karayilanoglu, Department of NBC Defence, Gulhane Military Medical Academy, Ankara, Turkey
} 


\section{REFERENCES}

1 Brennan RJ, Waeckerie JF, Sharp TW, et al. Chemical warfare agents. Emergency medical and emergency public health issues. Ann Emerg Med 1999;34:191-204.

2 Shemer J, Danon YL. Eighty years of the threat and use of chemical warfare. The medical-organizational challenge. Isr J Med Sci 1991;27:602-12.

3 Kawana N, Ishimatsu S, Kanda K. Psycho-physiological effects of the terrorist sarin attack on the Tokyo subway system. Mil Med 2001;166(suppl 12):23-6.

4 Romano JA Jr, King JM. Psychological casualties resulting from chemical and biological weapons. Mil Med 2001;166(suppl 12):21-2.

5 Waeckerie JF, Seamans S. Executive summary: developing objectives, content, and competencies for the training of emergency medical technicians, emergency physicians, and emergency nurses to care for casualties resulting from nuclear, biological, or chemical (NBC) incidents. Ann Emerg Med 2001;37:587-601.

6 Macintyre AG, Christopher GW, Eitzen E Jr, et al. Weapons of mass destruction events with contaminated casualties. JAMA 2000;283:242-9.

7 McKee CB, for the Battlebook Project Team. The medical NBC battlebook. USACHPPM Tech Guide 244, August 2000
8 Sidell FR, Takafuji ET, Franz DR. Medical aspects of chemical and biological warfare. Bethesda, MD: Office of the Surgeon General, 1997.

9 US Army Medical Research Institute of Chemical Defense. Medical management of chemical casualties handbook, 3rd edn. Aberdeen Proving Ground, MD: US Army Medical Research Institute of Chemical Defense, 2000 .

10 Sidell FR. What to do in case of an unthinkable chemical warfare attack or an accident. Postgrad Med J 1990;88:70-84.

11 Raber E, Jin A, Noonan K, et al. Decontamination issues for chemical and biological warfare agents: How clean is clean enough? Int J Environ Health Res 2001;11:128-48.

12 Kenar L, Karayilanoglu T, Kose S. Laboratory conditions and safety in a chemical warfare agent analysis and research laboratory. Mil Med 2002; 167:628-33.

13 Karalliedde L, Wheeler $\mathrm{H}$, Maclehose R, et al. Possible immediate and longterm health effects following exposure to chemical warfare agents. Public Health 2000; 114:238-48.

14 Cox RD. Decontamination and management of hazardous materials exposure victims in the emergency department. Ann Emerg Med 1994;23:761-70. 